\title{
La innovación urbana como factor de desarrollo socioeconómico ${ }^{1}$
}

\author{
Julio César Caro Moreno² \\ Universidad Pedagógica y Tecnológica de Colombia - UPTC \\ julio.caro@uptc.edu.co
}

DOI: https://doi.org/10.21158/01208160.n82.2017.1645

Fecha de recepción: 22 noviembre de 2016

Fecha de aprobación: 9 de mayo de 2017

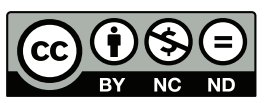

Cómo citar este artículo/ To reference this article/ Comment citer cet article/ Para citar este artigo:

Caro, J. C. (2017). La innovación urbana como factor de desarrollo socioeconómico.

Revista EAN, 82, pp.161-174. https://doi.org/10.21158/01208160.n82.2017.1645

\section{Resumen}

En el siguiente artículo, se revisa el planteamiento de los autores más representativos en la geografía urbana que desarrollan el enfoque de la innovación como un factor de desarrollo en las ciudades y sus actores. El objetivo es identificar cómo se incorpora el proceso de innovación en lo urbano como factor de desarrollo socioeconómico, tras lo cual se concluye que en la actualidad la relación innovación urbana y desarrollo está generando nuevas estructuras en cada ciudad que fomentan la inclusión en los cambios de todos los actores, que promueven los grupos tecnológicos a toda escala, estableciendo ciudades con gran avance tecnológico y procesos socioeconómicos clave para el desarrollo.

\section{Palabras clave}

Innovación urbana, geografía de lo urbano, desarrollo.

1 Este artículo es resultado del proyecto adscrito a la Dirección de Investigación de la Universidad Pedagógica y Tecnológica de Colombia.

2 Economista. Especialista en Finanzas. Magíster en Economía. Doctorando en Geografía. Docente de la Universidad Pedagógica y Tecnológica de Colombia. ORCID: http://orcid.org/0000-0001- 5470-6752 


\title{
Urban Innovation as a Socioeconomic Development factor
}

\begin{abstract}
This article reviews the studies made by different representative authors in the field of urban geography which propose an innovation focus as a growing factor in cities and their agents. It aims at identifying how to incorporate an innovation process in urban areas as a socioeconomic factor through which a relation between urban innovation and development generate new city restructuring to foster inclusion in all agent changes, promoting technological groups at all levels and establishing cities with huge technological advancement and key socioeconomic processes for development.
\end{abstract}

Key words. Urban innovation, urban geography, development.

\section{$\longrightarrow$ \\ L'innovation urbaine, un facteur de développement socio-économique}

Resumé. Cet article présente une révision des thèses des auteurs les plus représentatifs en matière de géographie urbaine qui voient en l'innovation un facteur de développement des villes et de leurs acteurs. Notre objectif est d'identifier comment le processus d'innovation s'incorpore dans l'environnement urbain en tant que facteur de développement socio-économique, grâce auquel la relation entre l'innovation urbaine et le développement génère de nouvelles structures dans chaque ville permettant l'augmentation des échanges entre les acteurs, confortant les groupes technologiques de tous les niveaux, modelant des villes à la pointe du changement technologique et des processus socio-économiques clefs pour le développement.

Mots clefs. Innovation urbaine, géographie urbaine, développement.

\section{$\longrightarrow$ \\ A inovação urbana como fator de desenvolvimento socioeconômico}

\begin{abstract}
Resumo. No seguinte artigo, se revisa a proposta dos autores mais representativos da geografia urbana que desenvolvem o enfoque da inovação como um fator de desenvolvimento nas cidades $e$ em seus atores. Seu objetivo é identificar como se incorpora o processo de inovação no urbano como fator de desenvolvimento socioeconômico. Conclui-se que atualmente a relação inovação urbana $e$ desenvolvimento está gerando novas estruturas em cada cidade, fomenta a inclusão de todos os atores nas mudanças, promove os grupos tecnológicos a toda escala, estabelecendo cidades com grande avanço tecnológico e processos socioeconômicos chaves para o desenvolvimento.
\end{abstract}

Palabras chave. Inovação urbana, geografia do urbano, desenvolvimento. 


\section{Introducción}

디 proyecto de investigación del que se cderiva este artículo se enmarca en la importancia que tiene el proceso de innovación en los territorios y regiones como eje de desarrollo socioeconómico desde una visión geográfica y otorga una marcada importancia a los actores territoriales y su respuesta al actual proceso de globalización, su problemática y retos económicos, sociales, ambientales, institucionales, entre otros, enmarcados en un territorio protagonista y dinámico en la construcción de su desarrollo.

Por lo anterior, en este artículo, se plantea revisar cómo algunos autores con enfoque en la geografía urbana incorporan la innovación como un factor de desarrollo de los territorios (ciudades) y sus actores o redes. Por lo que la pregunta por responder es esta: ¿cómo se incorpora el proceso de innovación territorial en la geografía urbana como factor de desarrollo?

Para dar respuesta a esta formulación, este artículo esboza, entre otros, una aproximación a la noción de territorio e innovación territorial como enfoque de la geografía urbana, el sistema urbano de innovación -milieu-, lo urbano como entorno innovador, aglomeraciones, innovación y ventajas competitivas, recursos generadores de un entorno innovador para el desarrollo, la ciudad como red estructurada de innovación $y$, finalmente, unas conclusiones.

\section{Aproximación a la noción de territorio y de innovación territorial: características y enfoques emergentes de la geografía urbana}

nn la actualidad, más de la mitad de la
población mundial es urbana y por tanto
mucho de lo que será el futuro de la sociedad
humana se desarrolla en las ciudades, por lo
que es importante impulsar la innovación
como proceso de desarrollo, ya que, como
se planteó en el World Urban Forum 7
(2014), la ciudad debe trascender la forma
y funcionalidad de los modelos anteriores
y equilibrar menores costos de energía con
una menor huella ecológica, una forma más
compacta y una mayor heterogeneidad. Al
mismo tiempo, esa nueva ciudad debe crear
condiciones para una provisión más elevada
de bienes públicos, junto con espacios más
creativos (innovadores) para la imaginación, la interacción social, así como es preciso reducir la vulnerabilidad.

En todo caso, en las grandes ciudades y áreas metropolitanas, se han estudiado las complejas estructuras que son reflejo de la estructura social y económica de una ciudad y los procesos de estructuración territorial en los que el uso del suelo es un factor esencial. Los urbanistas han aludido a la expansión continuada de la ciudad sobre el territorio circundante, para lo cual se han apoyado en las infraestructuras construidas y han denunciado la deterioración de las condiciones medioambientales, a la vez que la realización de gigantescos negocios 
inmobiliarios; el consumo de territorio se ha convertido en uno de los más relevantes negocios de la sociedad capitalista, por lo que autores como Sánchez-Hernández, Aparicio Amador y Alonso-Santos (2010, p. 23) muestran una preocupación por considerar la totalidad del espacio urbano, revelada a través de una orientación espacial e histórica de carácter territorial, que diese sentido al proceso urbano.

En el World Urban Forum 7 (2014), se planteó que los problemas urbanos esenciales son los causados por la insuficiencia de viviendas, las áreas de autoconstrucción y vivienda precaria, el acceso a servicios básicos como agua y desagüe, el suelo urbanizado bien localizado y los riesgos de desastres naturales, la falta de financiación adecuada para inversiones suficientes que permitan resolver la creación de empleo y los problemas existentes, la urgencia de conseguir un desarrollo con equidad y un desarrollo sostenible que minimice los riesgos naturales y el cambio climático.

También se afirma la necesidad del planeamiento, lo cual supone un cambio esencial frente a las políticas neoliberales que lo cuestionaban. Todo ello le da gran importancia a la innovación como proceso de desarrollo y paliativo a estas problemáticas.

La necesidad y urgencia de intervención en el territorio tras las crisis de los últimos dos decenios han conducido a numerosas propuestas de actuación que sean más integradoras y de carácter pluridisciplinario -algunas impulsadas por los economistas-, lo cual ha dado lugar al concepto de inteligencia territorial, es decir, a una actuación más inteligente sobre el territorio que tenga en cuenta los dinamismos territoriales, los recursos disponibles, las necesidades de las personas y los objetivos de una mayor equidad, la comunidad local (a veces calificada como comunidad territorial), y que tenga en cuenta, asimismo, a los agentes $y$ actores involucrados para forjar nuevas alianzas y concertaciones, y con ello nuevas y más eficaces estrategias locales de gestión. El concepto se aplica a diferentes escalas y a campos generales y específicos. Trata también de aplicar los avances, en especial, la innovación en la actual sociedad del conocimiento en favor de un desarrollo territorial (Sánchez-Hernández, AparicioAmador y Alonso-Santos, 2010, p. 23).

Echeverría y Merino (2011, p. 1) plantean que los estudios de innovación han experimentado un giro social que comienza a trasladarse a las políticas de innovación; estamos ante un nuevo paradigma en ese tipo de estudios, una ampliación de los tipos y modelos de innovación. Así, el desarrollo territorial debe fomentar procesos de innovación social, como el aprendizaje social, el diseño, la tecnología, las políticas públicas, las ciudades -innovación urbana-, los movimientos sociales y el desarrollo comunitario, por lo que concluyen que las tecnociencias de innovación social parecen haber llegado a un punto de amplio consenso ( $p$. 4): las innovaciones sociales surgen en primera instancia de la sociedad civil, pero también pueden ser generadas o implementadas por el sector público y por el sector privado. Además, tanto los medios como los fines para promoverlas han de ser predominantemente sociales; todo como desenlace de un mayor desarrollo socioeconómico en los territorios. 


\section{El sistema urbano de innovación: milieu}

A finales del siglo $X X$, surge un planteamiento que pretende explicar el cambio y la reestructuración económica y urbana y propone que los procesos de ajuste espacial y productivo obedecen, en buena medida, a factores de carácter endógeno, y recogen la respuesta de las empresas y los actores locales a los desafíos de la competitividad.

Esta corriente se denomina milieu, la cual permite definir los términos y las condiciones bajo las que los sistemas productivos locales

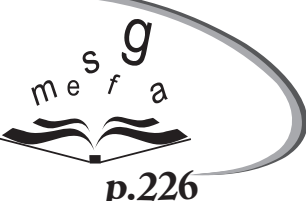

y las ciudades son capaces de dar las respuestas autónomas y estratégicas que les permiten mantener su posición en los mercados y en los sistemas urbanos. Los distritos y las ciudades son milieus, caracterizados por la capacidad organizativa y la dinámica de aprendizaje de sus actores. El éxito en los procesos de ajuste depende del carácter innovador de ambos milieus y de la sinergia y cooperación que se puede establecer entre ellos (Vázquez-Barquero, 1999, p. 2).

El concepto de milieu incorpora los avances que ha introducido la teoría espacial del desarrollo al rescatar el papel que desempeñan el territorio y la innovación en la dinámica productiva. El territorio no sería el soporte físico de los recursos, de las actividades productivas y de las relaciones económicas y sociales. Como lo plantea Vázquez-Barquero (1999, p. 3), el territorio sería, más bien, el lugar en el que los agentes económicos, sociales, culturales y políticos se organizan, utilizan sus capacidades y conocimientos y producen e intercambian recursos, bienes y servicios.
Consecuentemente, Maillat (1992, p. 41) plantea que la corriente milieu está formada por un sistema de agentes y elementos económicos, socioculturales, políticos e institucionales que poseen modos de organización y regulación específicos. También considera que los milieus locales son incubadoras de las innovaciones urbanas, ya que la empresa hace parte fundamental de un milieu, la innovación depende de la organización del territorio, de la interacción de los agentes, por consiguiente, de la historia concreta del lugar.

Maillat (1992, p. 16), expone que las estructuras de producción locales en las ciudades logran conceptualizarse como una estructura innovadora milieu que se caracterizaría por una estructura de organización y una dinámica de aprendizaje, consiente a las dinámicas cambiantes del entorno.

Los milieus urbanos son una estructura en la que los agentes e instituciones locales tienen relación e intercambio en la economía de bienes, servicios y del saber hacer, de acuerdo con las reglas fijadas. Según su dinámica, se plantea una constante trasformación y de ella un aprendizaje, que permite la investigación y el desarrollo que pretende crear innovaciones de cada actor, su cooperación y la formación de redes entre ellos.

Los milieus definen que la ciudad es un territorio construido, con fronteras no bien definidas, al que pueden aplicarse los paradigmas cognoscitivo, organizativo y territorial. Las ciudades se diferencian, por tanto, unas de otras en función de las carac-terísticas que las definen y solo aquellas en las que se organizan los procesos de innovación pueden identificarse como milieu innovador (VázquezBarquero, 1999, p. 4). 


\section{Lo urbano como entorno innovador}

M éndez, García y Michelini (2007, p. 5) describen la importancia del proceso innovador en ciudades intermedias para generar, difundir e intercambiar conocimiento y aprovechar sus recursos patrimoniales, que así como con la construcción de redes locales de actores y su inserción en redes supralocales permiten la consolidación de entornos innovadores, capaces de promover no tanto mayor crecimiento como mejor desarrollo. Se trata, pues, de un proceso de construcción de ciudad que desborda la dimensión económica para incorporar la sociocultural y la institucional.

Méndez, García y Michelini (2007) soportan su planteamiento teórico en el medio innovador del Groupe de recherche européen sur les milieux innovateurs (GREMI), en sus intentos por comprender por qué ciertos lugares son capaces de promover la producción e incorporación de innovaciones generadoras de ventajas competitivas.

Por su parte, la Unesco (2010) define las learning cities como aquellas que tienen en común su voluntad de situar la innovación y el aprendizaje en el centro de su estrategia de desarrollo, al combinar el aprendizaje continuo, la innovación y la utilización creativa de las tecnologías de la información y la comunicación como claves de su dinamismo. La acumulación de capital social asociado a la presencia de redes locales, y el papel esencial de las instituciones en su doble sentido de convenciones, normas y valores por una parte, y organizaciones sociales por otra, son la clave para su construcción.

Es muy importante el proceso de construcción del territorio con sus propios recursos (Méndez, García y Michelini, 2007, p. 7). Así, la ciudad se entiende como construcción social donde, a partir de unas dotaciones iniciales variables, algunas sociedades son capaces de generar un escenario favorable a la innovación mediante la movilización de recursos específicos que hacen posible la creación de unas condiciones solo entendibles en relación con ese ámbito.

Entre las principales dotaciones, están las que deben convertirse en activos movilizados por la sociedad local (Colletis y Pecqueurb, 1995). Aquí se encuentran los infraestructurales -servicios avanzados, equipamientos para la innovación, centros educativos-, los humanos -población con cierto nivel formativo, el saber hacer local-, los productivos -estructura empresarial heredada, cultura empresarial- o los patrimoniales. Pero se considera de especial importancia el marco institucional, directamente ligado a la noción de capital social (Camagni, 2003) (Figura 1). 
Figura 1. Ciudades innovadoras y desarrollo local: factores y componentes.

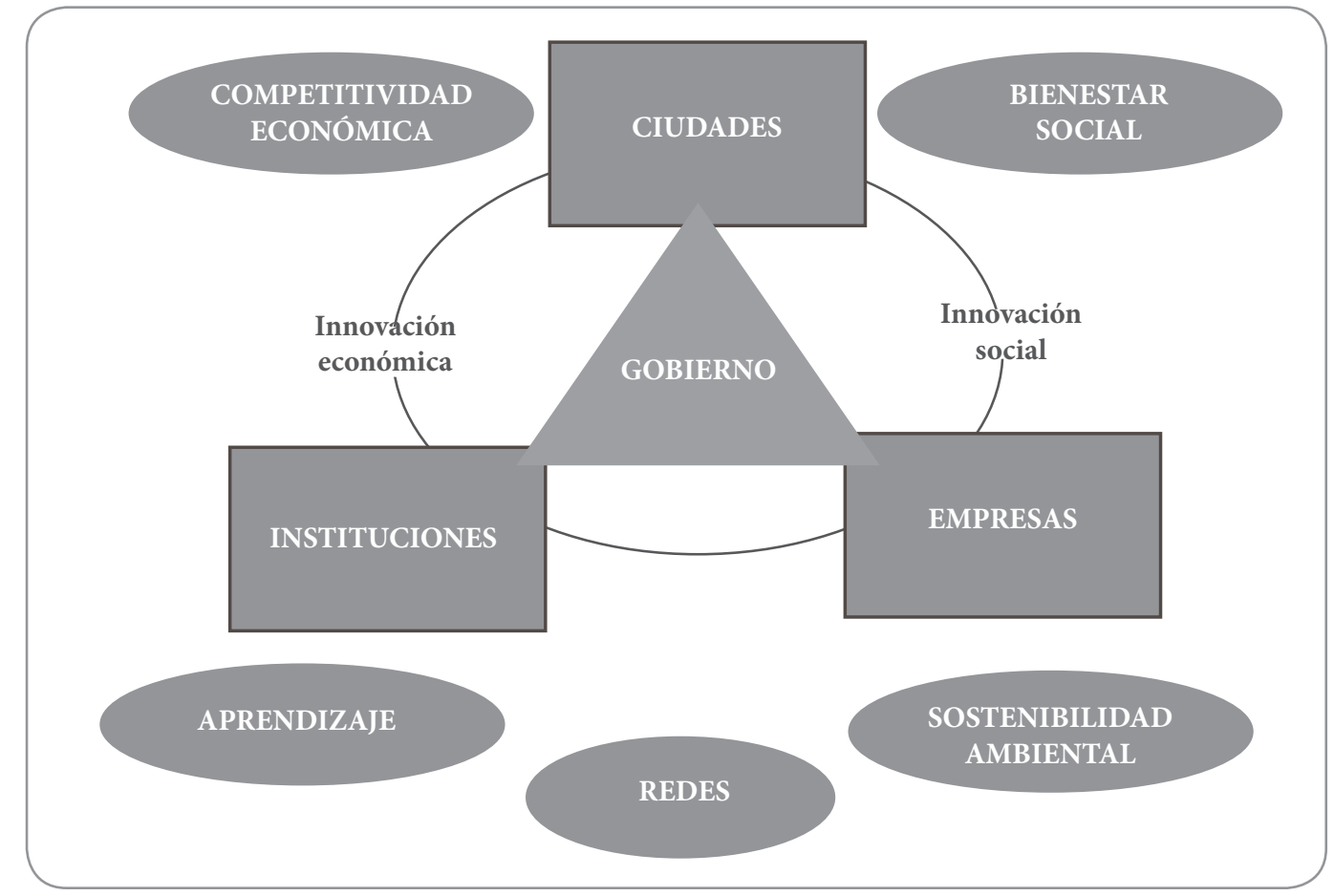

Fuente. Elaboración propia según Méndez, García y Michelini, (2007, p. 7).

Esta nueva manera de interpretar el dinamismo urbano y su capacidad de inducir el desarrollo territorial señala, que para comprender los procesos de innovación en el seno de las empresas y de los sistemas productivos, hay que trascender ese ámbito de investigación a fin de fijarse en la posible existencia de redes sociales e institucionales de apoyo.
Así pues, para intensificar los procesos de aprendizaje colectivo, es necesario que los múltiples integrantes de los sistemas locales de innovación con especial protagonismo de los Gobiernos locales establezcan ciertas relaciones de cooperación, lo cual exige la creación de un clima de confianza, generalmente difícil de alcanzar (Méndez, García y Michelini, 2007). 


\section{Procesos de metropolización, redes de ciudades y aglomeraciones}

Transformaciones que se asocian de forma directa a nuevas formas urbanas, que han dado origen a una amplia proliferación de neologismos y metáforas en los últimos años, que se superponen a los de metrópolis, conurbación o megalópolis, de más larga tradición, y ponen de manifiesto las dificultades del lenguaje para proporcionar instrumentos capaces de describir adecuadamente la nueva urbe. (Amendola, 2000, p. 32).

En unos casos, hacen referencia a su contenido funcional y al protagonismo de algunas ciudades en la era del capitalismo global -ciudad posindustrial, global, mundial-, mientras en otros destacan la relación entre nuevo marco tecnológico y estructura urbana -ciudad posfordista, informacional, digital, telépolis, ciberciudad, softcit-, el reforzamiento de los contrastes internos -ciudad collage, ciudad fractal, ciudad dualo suponen la integración de algunas dentro de la llamada

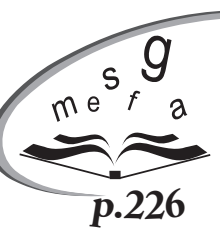
sociedad del conocimiento - ciudad innovadora, inteligente, creativa- (Méndez, 2007, p. 3).

De acuerdo con lo anterior, es evidente que las ciudades, $y$ en especial las de mayor tamaño, tienen unas actuaciones innovadoras que se diferencian y que se caracterizan por su territorialidad, como desarrollo histórico; esto se incorpora en el análisis de la actividad económica metropolitana, lo cual supone hacer complementarias y compatibles dos tipos de argumentación (Figura 2).

Figura 2. Ventajas competitivas y procesos de desarrollo local en espacios metropolitanos.

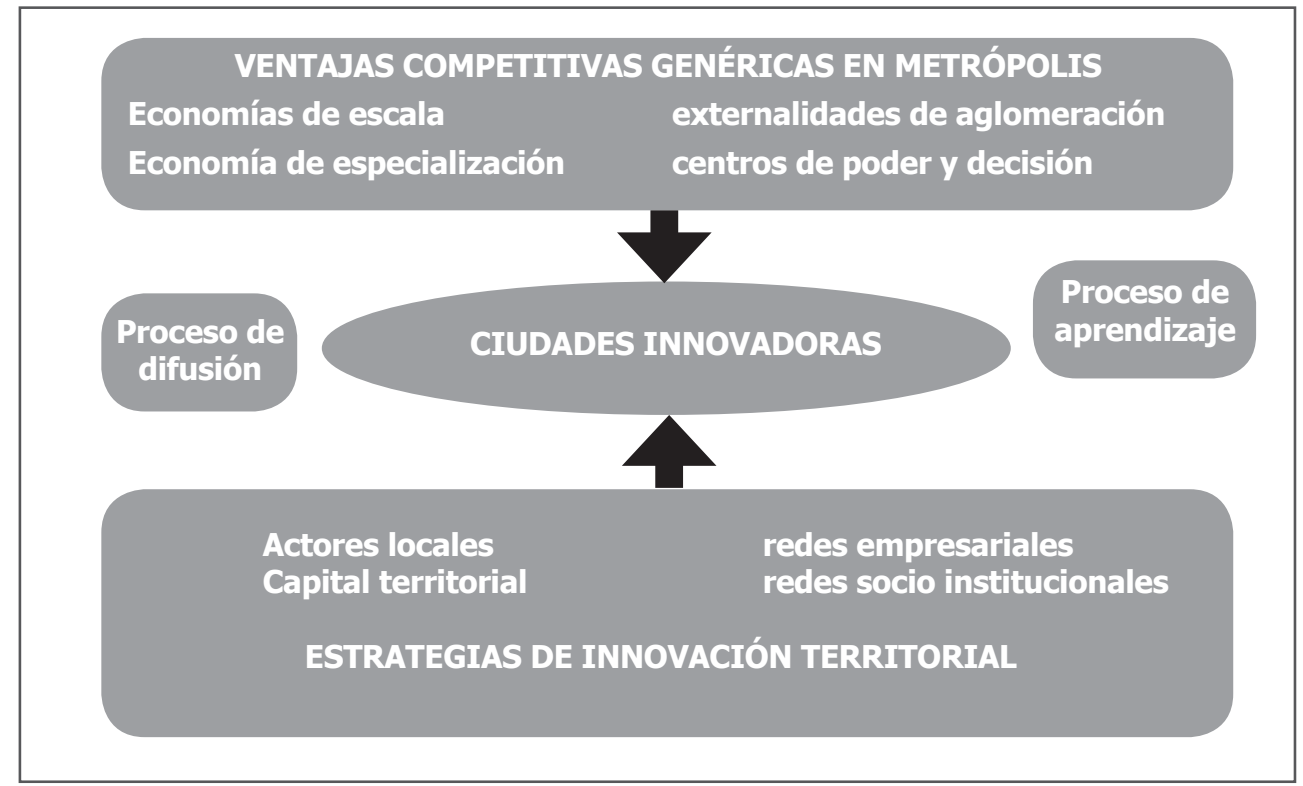

Fuente. Elaboración propia según Méndez, (2007, p. 11). 
Por su parte, Camagni (2003) plantea que, desde una perspectiva de economía urbana, los argumentos explicativos de las ventajas competitivas de las grandes ciudades y las aglomeraciones urbanas concedieron siempre especial importancia al efecto derivado de la dimensión que, además de generar economías de escala derivadas de la indivisibilidad de algunos procesos, sería la causa fundamental de la aparición de externalidades positivas.

Según esta interpretación, una gran cantidad de regiones metropolitanas del mundo serán las que, con su acopio de capital físico, humano, financiero y de conocimiento, generan el mayor volumen de economías de localización y urbanización, lo cual es un atrayente para todo tipo de actividades, principalmente las de alto contenido de ciencia, tecnología e innovación.

A partir de las grandes concentraciones en la ciudad, algunas de esas actividades podrán luego difundirse de forma progresiva y limitada hacia ciertas ciudades del entorno próximo e intermedio que crearan lo que se denomina un sistema urbano. Eso convierte a las aglomeraciones metropolitanas en potenciales ambientes innovadores, pese a que la activación de ese potencial resulta claramente distinta según los casos (Méndez, 2007).

\section{Recursos generadores de un entorno innovador para el desarrollo}

$T^{\circ}$ odas estas iniciativas comparten la lógica de la visión territorial del desarrollo, y, en particular, la de la interpretación del desarrollo natural (Pyke, Rodríguez y Tomaney, 2006, p. 51). Si se acepta que el desarrollo está enraizado en el territorio, parecería lógico considerar que es el resultado de combinar en los procesos productivos los recursos naturales, la fuerza de trabajo local, la tecnología y el conocimiento acumulados en las empresas y las organizaciones, así como la capacidad emprendedora existente en el territorio.

La adecuada combinación de políticas y recursos en la planeación y dinámica de las ciudades produce un efecto favorable en el bienestar económico y social de la población que vive en el territorio. Esta interpretación señala la importancia de los factores geográficos en los procesos de desarrollo económico, y por tanto, como sostiene Sachs (2011, p. 141), la dinámica económica de las localidades y ciudades de las economías emergentes y de desarrollo tardío se podría analizar en función de los factores geográficos y ecológicos que afectan las actividades productivas, la salud y los recursos.

Para que se dé un proceso de innovación y por ende un impacto de desarrollo socioeconómico de los territorios, se debe generar una presencia e interacción de los actores y los recursos del territorio como generadores de un sistema económico propio, que responda a las necesidades propias y articule a todos los agentes -crea o activa redesque actúan en proximidad como ventaja geográfica (Figura 3). 
Figura 3. Condiciones para la formación y el desarrollo de un medio o entorno innovador.

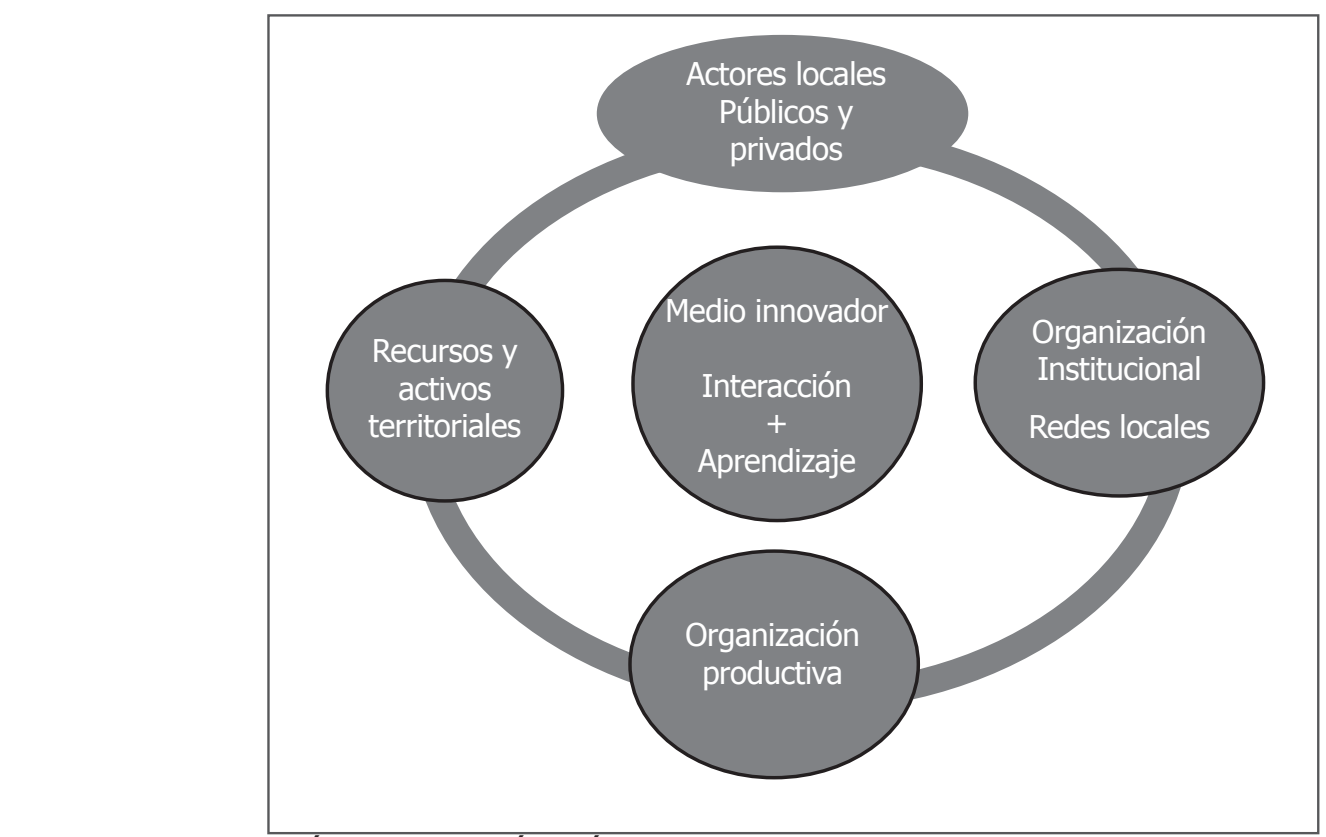

Fuente. Elaboración propia según Méndez, (2002, p. 14).

El medio innovador es reflejo del aprendizaje y de la relación interactiva de los actores, un territorio que genera un proceso de innovación de un sistema local o red de agentes vinculados por lazos sociales -con fianza- y económicos -transacciones-construidos a lo largo del tiempo en un marco territorial definido de escala global o local.

Los agentes económicos operan en un tiempo histórico cuando el aprendizaje, fruto de las decisiones pasadas, orienta su labor cotidiana, lo cual refuerza el valor de la confianza como condicionante de los procesos económicos y muy en particular de la innovación, siempre sujeta a riesgos solo compartidos entre quienes se reconocen mutuamente como miembros de una comunidad. De ahí que la acumulación de transacciones mercantiles no sea suficiente para transformar un sistema local de empresas en un medio innovador: también es preciso constituir una red de agentes enfocada al aprendizaje colectivo (Sánchez-Hernández, Aparicio-Amador y Alonso-Santos, 2010).
El soporte de los actores locales en el proceso innovador y de desarrollo socioeconómico es fundamental en especial en las alianzas de instituciones públicas y privadas que en algunos lugares resultan un eficaz complemento al esfuerzo de innovación empresarial (Alonso y Méndez, 2000, p. 20) (Figura 4).

Las instituciones públicas son todas las autoridades y administraciones que tienen poder sobre el territorio y un marco descentralizado -territorial-, por ende, actores y conocedores del enfoque de desarrollo propio, lo cual las hace protagonistas de primera línea; también las instituciones nacionales, internacionales y supranacionales tienen un papel en el proceso innovador y de desarrollo. Las instituciones privadas, entre ellas, las empresas, las organizaciones, los centros tecnológicos, entre otras, actúan como dinamizadoras de iniciativas colectivas de carácter local. 
Figura 4. Actores para la innovación y el desarrollo territorial.

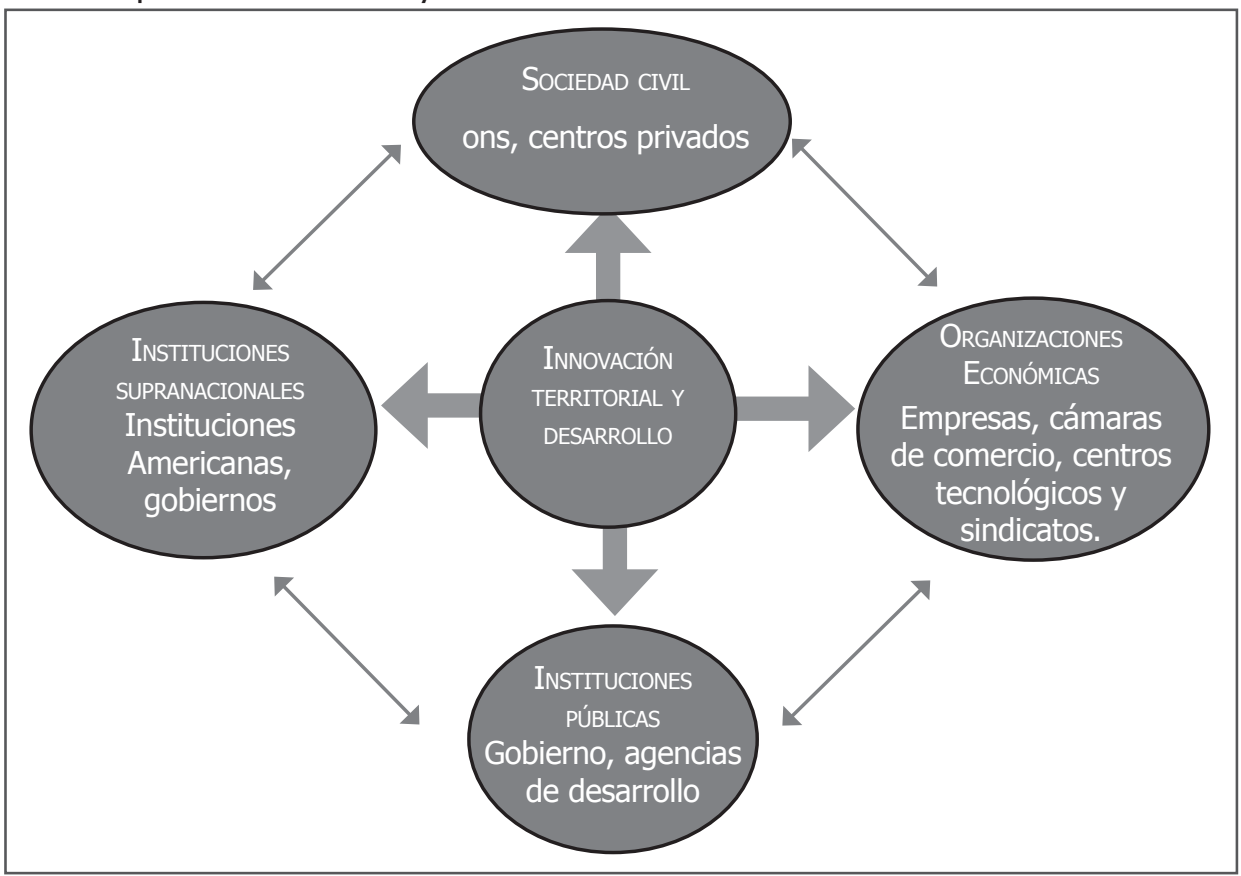

Fuente. Elaboración propia según Alonso y Méndez, (2000, p. 20).

\section{La ciudad como red estructurada de innovación}

n la actualidad, el proceso de descubricmiento y generación de nuevas estructuras sociales de innovación se plantea en forma de comunidades abiertas, también

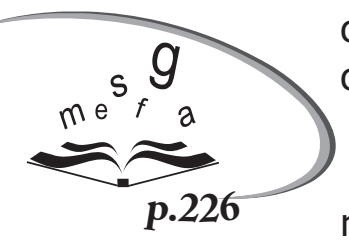
conocidos como laboratorios ciudadanos. Cuando se habla de comunidades abiertas de innovación, se hace referencia tanto a sectores económicos como a territorios, ciudades o distinto tipo de comunidades, que abren la innovación a todos los actores y agentes y así generan nuevas oportunidades socioeconómicas.

El surgimiento en las ciudades de grupos innovación parece estar en la base de nuevos modos de socialización de las tecnologías. Además, en algunos casos, en los territorios, dichas tecnologías no están necesariamente desde el inicio, por lo que es el territorio el generador de procesos de innovación social abierta y dirigida por los ciudadanos, lo cual genera una conexión e interacción entre las instituciones públicas, las comunidades y las formas de innovación emergente como proceso de desarrollo (Schiavo y Serra, 2013, p. 4).

Por su parte, Dupuy (1998), planteó una mirada dinámica de lo urbano, focalizada en las redes de infraestructura o redes técnicas urbanas. Dicha perspectiva visualiza las redes como espacios en sí mismos, tanto material como imaginariamente. Además, Castells (2001) añadió la visión de una ciudad internet, lo cual lo conduce a plantear un nuevo modelo de ciudad dual y una nueva categoría de incluidos/excluidos en función de la red digital global. Lo anterior plantea que se debe ver la ciudad como una red estructurada y no como una organización solo reticular. 


\section{Conclusión}

다 objetivo de este artículo fue revisar el - planteamiento de algunos autores sobre la innovación territorial en la geografía urbana como factor de desarrollo socioeconómico. Inicialmente, se identifica la importancia que tiene lo urbano, en especial la ciudad como medio de innovación territorial, al proveer todo tipo de bienes y espacios creativos -uso del suelo-, como generador de desarrollo socioeconómico para los que habitan estas urbes.

El proceso de innovación urbano es más acelerado y se explica por la aglomeración y las problemáticas que genera y que corresponde resolver de manera ingeniosa y sostenible, ya que se forma en la ciudad una inteligencia territorial que muestra que existen dinámicas entre los actores locales y su territorio como proceso de desarrollo, en el que tanto los medios como los fines han de ser predominantemente socioeconómicos.

Los distritos y las ciudades son milieus, caracterizados por la capacidad organizativa y la dinámica de aprendizaje de sus actores. Este incorpora los avances que ha introducido la teoría espacial del desarrollo al rescatar el papel que desempeñan el territorio y la innovación en la dinámica productiva. Otro concepto es las ciudades que aprenden: que planean su desarrollo al combinar el aprendizaje continuo, la innovación y la utilización creativa de las tecnologías de la información y la comunicación como claves de su desarrollo.

Las nuevas formas urbanas, su tamaño y dinámica hacen que cada territorio tenga su propia dinámica de innovación, lo cual permite una competitividad que caracteriza su actividad y desarrollo a toda escala; esta innovación puede ser difundida en un entorno próximo e intermedio y crear potenciales ambientes innovadores para el desarrollo económico y social de la sociedad que vive en un territorio, lo cual constituye una ventaja geográfica.

La participación de los actores locales en el proceso innovador y de desarrollo es fundamental, ya que son estos los que conocen las problemática en el territorio; esto crea redes o alianzas entre las instituciones públicas, privadas y las empresas, y en algunos lugares, resulta un eficaz complemento al esfuerzo de innovación territorial.

La actual relación innovación territorial y desarrollo socioeconómico está generando nuevas estructuras en cada localidad que fomentan la inclusión de los cambios de todos los actores que promueven los grupos tecnológicos a toda escala (pública, privada, mixta), como forma de innovación emergente, que crea ciudades con perfil global (redes técnicas urbanas), por su avance tecnológico y de comunicación en algunos procesos socioeconómicos, claves para el desarrollo.

Finalmente, se propone crear redes que favorezcan el proceso de innovación urbana y que fomenten el conocimiento, pero se debe partir del entendimiento de que la innovación es dinámica y de que tiene un proceso de incorporación y aplicación a las necesidades económicas, sociales, culturales, políticas y ambientales, las cuales deben generar beneficios al mayor rango posible de personas, grupos e instituciones $y$, por ende, promuevan un desarrollo de los espacios urbanos. 


\section{Referencias}

Alonso, J. L. y Méndez, R. (coords.) (2000). Innovación, pequeña empresa y desarrollo local en España. Civitas.

Aparicio, J., Sánchez, J. L., Alonso, J. L. y Rodero, V. (2008). La Ribera del Duero, geografía de un medio innovador en torno a la vitivinicultura. Scripta Nova. Revista Electrónica de Geografía y Ciencias Sociales, 12(277). Recuperado de http:// www.ub.edu/geocrit/sn/sn-277.htm

Amendola, G. (2000). La ciudad postmoderna: magia y miedo de la metrópolis contemporánea. Madrid: Celeste.

Camagni, R. (2003). Incertidumbre, capital social y desarrollo local: enseñanzas para una gobernabilidad sostenible del territorio. Investigaciones Regionales, 2, 31-57.

Capel, H. (2016). Las ciencias sociales y el estudio del territorio. Revista Bibliográfica de Geografía y Ciencias Sociales, 21(1149), 742-798.

Castells, M. (2001). Internet y la sociedad red. La Factoría, 14. Recuperado de https://goo.gl/92M3FQ

Colletis, G. y Pecqueur, B. (1995). Politiques technologiques locales et création de ressources spécifiques. En A. Rallet y A. Torre, Economie industrielle et économie spatiale (pp. 445-463). París: Economica.

Dupuy, G. (1998). El urbanismo de las redes: teorías y métodos. Barcelona: Oikos-Tau.
Echeverría Ezponda, J. y Merino Malillos, L. (2011). Cambio de paradigma en los estudios de innovación: el giro social de las políticas europeas de innovación. ARBOR Ciencia, Pensamiento y Cultura, 187(752), 1031-1043.

Maillat, D. (1992). La relation des entreprises innovatrices avec leur milieu. En D. Maillat y J.-C. Perrin (eds.), Entreprises innovatrices et développement territorial (pp. 3-22). GREMI/EDES. Recuperado de http://wwwa.unine.ch/irer/gremi/ Gremi\%202.pdf\#page=7

Méndez, R. (2002). Innovación y desarrollo territorial: algunos debates teóricos recientes. EURE, 28(84), 63-83.

Méndez, R. (2007). El territorio de las nuevas economías metropolitanas. Eure, 33(100), 51-67.

Méndez, R., García Palomares, J. C. y Michelini, J. J. (2007). La construcción de ciudades innovadoras: transformaciones económicas recientes y desarrollo local en Getafe. Estudios Geográficos, 68(262), 173-203.

Pyke, A., Rodríguez Pose, A. y Tomaney, J. (2006). Local and regional development. Londres: Routledge.

Sachs, D. J. (2011). Tropical underdevelopment. Cambridge MA.: National Bureau of Economic Research.

Sánchez-Hernández, J. L., Aparicio-Amador, J. y Alonso-Santos, J. L. (2010). The shift between worlds of production as an 
innovative process in the wine industry in Castile and Leon (Spain). Geoforum, 41(3), 469-478.

Schiavo, E. y Hurtado, A. S. (2013). Laboratorios ciudadanos e innovación abierta en los sistemas CTS del siglo XXI: una mirada desde Iberoamérica: presentación. CTS: Revista Iberoamericana de Ciencia, Tecnología y Sociedad, 8(23), 115-121.

Unesco (Organización de las Naciones Unidas para la Educación, la Ciencia y la Cultura) (2010). Pilot Workshop on Developing Capacity for Establishing Lifelong.
Hamburgo: Learning Systems in UNESCO Member States. Recuperado de http:// uil.unesco.org/fileadmin/keydocuments/ LifelongLearning/en/Final\%20Report CapLLL_Pilot_Workshop_22_02_2011.pdf

Vázquez-Barquero, A. (1999). Dinámica productiva y desarrollo urbano: la respuesta de la ciudad de Vitoria (País Vasco) a los desafíos de la globalización. EURE, 25(74), 19-33.

World Urban Forum 7, Medellín, 5 al 11 de abril 2014. Recuperado de http://wuf7. unhabitat.org/theworldurbanforum-es 